\title{
Typologies of postnatal support and breastfeeding at two months in the UK
}

\author{
Emily H. Emmott ${ }^{\mathrm{a}, *}$, Abigail E. Page ${ }^{\mathrm{b}}$, Sarah Myers ${ }^{\mathrm{a}}$ \\ ${ }^{a}$ UCL Anthropology, University College London, 14 Taviton Street, London, WC1H OBW, UK \\ ${ }^{\mathrm{b}}$ Department of Population Health, London School of Hygiene and Tropical Medicine, Keppel Street, London, WC1E 7HT, UK
}

A R T I C L E I N F O

\section{Keywords:}

Breastfeeding

Mothers

Infant feeding

Social support

Latent class analysis

UK

\begin{abstract}
A B S T R A C T
There is extensive evidence to suggest that social support improves breastfeeding outcomes. Building on this evidence-base, public health services and interventions aiming to improve breastfeeding rates have primarily targeted informational and emotional support to mothers, reflecting an individual behaviour-change approach. However, mothers exist within a wider social network, and the characteristics of their broader support networks may be an important predictor of breastfeeding outcomes. Here we explore the typologies of postnatal support for mothers in the UK; a population with one of the lowest breastfeeding rates in Europe. Using retrospective data from an online survey (data collection period December 2017 - February 2018), we carry out a latent class regression $(n=432)$ to identify "clusters" of postnatal support in our data. Mothers in our sample were most likely to report receiving practical and emotional support from partners and maternal grandmothers, and breastfeeding information from health professionals. We identify three distinct typologies of postnatal support: 1) Extensive support, where mothers received support from a wide range of supporters including partners, maternal grandmothers, friends and health professionals, but mothers were the only ones to feed the infant; 2) Family support, where mothers received support from partners and maternal grandmothers, including with infant feeding, but less likely to receive support from health professionals; and 3) Low support, where mothers primarily received support from partners. 94\% of women with extensive support were predicted to be breastfeeding at two months, followed by $48 \%$ of mothers in the low support group, and $13 \%$ in the family support group. Our findings highlight the complexities of family support and its potential impact on breastfeeding, as well as the significance of professional support. Overall, our results hint at the potential value for health professionals to engage with wider family in order to achieve extensive support for mothers.
\end{abstract}

\section{Author contributions section}

Emily H Emmott: Conceptualization; Data curation; Formal analysis; Investigation; Methodology; Project administration; Validation; Visualization; Roles/Writing - original draft; Writing - review \& editing. Abigail E Page: Conceptualization; Data curation; Investigation; Project administration; Validation; Writing - review \& editing. Sarah Myers: Conceptualization; Investigation; Project administration; Validation; Writing - review \& editing.

\section{Introduction}

Breastfeeding has been associated with numerous physical health benefits for both mother and baby (Allen and Hector, 2005; Victora et al., 2016; Kramer and Kakuma, 2012), and the World Health Organisation currently recommends exclusive breastfeeding for 6 months (Victora et al., 2016; Kramer and Kakuma, 2012). However, despite continued efforts from the public health community (Bosi et al., 2016; Labbok, 2012), breastfeeding rates remain low in many developed countries (Bosi et al., 2016). In particular, breastfeeding rates in the UK are among the lowest in Europe (Bosi et al., 2016; UNICEF, 2013): A 2010 government survey estimated that $1 \%$ of UK women breastfeed exclusively at 6 months (Bosi et al., 2016; McAndrew et al., 2012), which is notably low compared to, for example, $18 \%$ in Netherlands and $28.5 \%$ in Spain (Bosi et al., 2016). In 2017/18, 42.7\% of mothers in England were estimated to be providing breastmilk at 6-8 weeks (Public Health England, 2018a). In the aforementioned 2010 survey, around $80 \%$ of UK women who stopped breastfeeding within the first 6 weeks said they would have liked to breastfeed for longer (McAndrew et al., 2012), highlighting high levels of "unmet feeding goals." Studies have repeatedly shown that such unmet feeding goals are often accompanied by a sense of guilt and failure (Burns et al., 2010), and problems associated with breastfeeding have been associated with postnatal depression (Brown et al., 2016).

\footnotetext{
* Corresponding author.

E-mail address: emily.emmott@ucl.ac.uk (E.H. Emmott).
} 
Most mothers in the UK seem to share goals with the broader public health community in that they want to breastfeed, and breastfeed for longer. Why, then, are so many mothers currently struggling to meet their feeding goals? Studies suggest that the reasons for early breastfeeding cessation are multi-faceted and complex (Thulier and Mercer, 2009), spanning individual circumstance as well as socio-cultural factors. Many Western countries have a recent history of predominant formula feeding (Fomon, 2001), meaning the cultural norms for breastfeeding have been weakened. Many mothers are unable to draw on adequate breastfeeding support from family, friends, and sometimes from health professionals; and in some cases are discouraged by them from breastfeeding (Hoddinott and Pill, 1999; Fox et al., 2015; Taylor et al., 2019). Further, studies suggest mothers are often underprepared for the challenges of breastfeeding due to unrealistic messaging: Breastfeeding in the West is often promoted as being "natural," which can lead to misconceptions that it is an instinctive/easy behaviour (Fox et al., 2015; Brown, 2016; Martucci and Barnhill, 2018; Williamson et al., 2012) - when in fact many women find breastfeeding challenging (Fox et al., 2015; Taylor et al., 2019; Brown, 2016; Williamson et al., 2012; Scott and Colin, 2002; Guyer et al., 2012). Overall, the lack of practical breastfeeding knowledge, combined with the shortage of breastfeeding support, leaves mothers vulnerable to breastfeeding challenges (Fox et al., 2015).

In response to such findings, public health services and interventions have focused on providing breastfeeding support, typically entailing provision of advice and information to mothers via health professionals or trained peer supporters (McFadden et al., 2017; Emmott and Mace, 2015). A recently updated Cochrane review of breastfeeding interventions found that support from health professionals and trained peers was associated with increased breastfeeding duration (McFadden et al., 2017). However, in the UK, the majority of randomised control trials (RCTs) have been ineffective at increasing breastfeeding initiation or duration (Hoddinott et al., 2011), and a systematic review of peersupport RCTs did not find evidence that such interventions improved breastfeeding outcomes (Jolly et al., 2012). While there are multiple causes leading to unsuccessful interventions (Hoddinott et al., 2011), we note that the current public health approach around breastfeeding typically focuses on a narrow pool of individuals as supporters, usually health professionals or trained peers, and sometimes fathers (Emmott and Mace, 2015). Interventions and policies in the UK are generally designed around individual behaviour change (Hoddinott et al., 2011), meaning they primarily target the mother. These approaches overlook the fact that mothers exist within a wider social network where friends and family influence maternal attitudes, knowledge, and experience of breastfeeding (Hoddinott and Pill, 1999; Fox et al., 2015; Emmott and Mace, 2015; Clifford and McIntyre, 2008; Schafer et al., 2016; Lavender et al., 2006). The core aim of our study is to broaden the focus of breastfeeding support to include wider family and friends, as well as health professionals, and examine its associations with breastfeeding outcomes in the UK.

\subsection{The importance of wider support}

Taking an evolutionary anthropological approach, we conceptualise breastfeeding as a "costly" maternal-investment behaviour (Emmott and Mace, 2015): Exclusive breastfeeding is estimated to require 450-700 kcals a day (Butte and King, 2005), increasing maternal nutritional requirements (Marlowe, 2003). Prolonged infant carrying, which often goes hand-in-hand with on-demand feeding, can be as energetically expensive as breastfeeding itself (Wall-Scheffler et al., 2007). On top of these energetic costs, breastfeeding often conflicts with other maternal activities leading to high opportunity costs (Emmott and Mace, 2015; Emmott et al., 2019; Quinlan and Quinlan, 2008; Hawkins et al., 2007; Cardenas and Major, 2005). For example, breastfeeding often conflicts with maternal labour: In developed populations, full-time maternal employment has been associated with a higher risk of breastfeeding cessation compared to part-time employment (Hawkins et al., 2007; Cardenas and Major, 2005; Johnston and Esposito, 2007; Fein et al., 2008). This conflict between breastfeeding and maternal labour is also observed cross-culturally, including in natural-fertility and subsistence populations (Emmott et al., 2019; Quinlan and Quinlan, 2008). In such populations, mothers are unable to simultaneously breastfeed and adequately provide for themselves and their infant on their own (Emmott et al., 2019; Quinlan and Quinlan, 2008). Consequently, mothers receive extensive support from a range of sources - including fathers, grandparents, aunts, uncles, and many nonrelatives, leading to a communal childrearing system (Emmott et al., 2019; Quinlan and Quinlan, 2008; Du et al., 2019; Page et al., 2017).

While the childrearing systems in developed populations have arguably shifted from communal care to intensive parenting (Faircloth et al., 2014), we nonetheless hypothesise that mothers in the West require extensive support from a wide range of individuals for successful breastfeeding. This has, to some extent, been evidenced in qualitative studies of maternal experiences of breastfeeding support. For example, focusing specifically on the UK, in a study of 23 mothers and their families in North-West England, mothers discussed the lack of wider family support as a barrier to breastfeeding, while acknowledging the benefits of support from partners, family and friends (Lavender et al., 2006). Similarly, in a study of mothers attending Breastfeeding Cafés across England, mothers discussed how the views and actions of health professionals, friends and family all facilitated or undermined breastfeeding (Fox et al., 2015). These realities and experiences of mothers indicate that the availability of support from a broad range of individuals may be an important determinant of breastfeeding outcomes in developed populations.

However, at present, very little is known around the wider support networks of mothers in the UK: Available quantitative studies often take a dyadic approach to breastfeeding support, where a specific supporter, usually a health professional or a trained peer supporter, delivers breastfeeding information and advice to the mother (McFadden et al., 2017). While qualitative studies have identified a range of individuals as important sources of support, studies tend to focus on understanding the meanings and consequences behind instances of support, rather than build up a picture of the broader support system. While such studies are highly valuable, we are yet to develop a good understanding of the characteristics of maternal support networks in the UK, and how these associate with breastfeeding outcomes. From a public health perspective, for breastfeeding support initiatives to be maximally effective, they must complement and work with the existing support system around mothers. A crucial step is therefore to understand who supports mothers with infants, and whether there are any systematic patterns in the sources of support.

\subsection{The importance of the types of support}

An additional point to consider alongside who helps is how individuals help mothers. In social epidemiology, social support has been broadly conceptualised as a "resource transfer" from one person to another (Stansfeld et al., 2006). Such support has been categorised into emotional and informational support, where individuals are provided with information and encouragement which improves their skill, selfappraisal and self-esteem, and practical or instrumental support, where individuals are supported via direct or tangible actions (Stansfeld et al., 2006). While there is theoretical recognition that different types of support may lead to different health and behavioural outcomes (Stansfeld et al., 2006), studies rarely define or clarify the types of support being investigated. Social support is often described as complex and multifaceted (McFadden et al., 2017; Hoddinott et al., 2011), but the majority of studies focus on informational and/or emotional support, meaning our current understanding of practical support and breastfeeding outcomes is limited (Emmott and Mace, 2015). From an evolutionary anthropological perspective, we hypothesise that the 
different types of support - informational, emotional and practical will have different pathways and effects on maternal breastfeeding (Tully and Ball, 2013):

1. Informational support involves knowledge transfer about infant feeding from the supporter to the mother. This may encourage breastfeeding via breastfeeding promotion, where discussion and knowledge of breastfeeding alters maternal perceptions of breastfeeding, normalising the activity (Emmott and Mace, 2015). Informational support may also increase maternal breastfeeding skill, thereby reducing the costs associated with breastfeeding. Note, the positive association between informational support and breastfeeding is contingent on the information being accurate, useful and helpful: Studies suggest conflicting and inaccurate information can undermine breastfeeding (Fox et al., 2015; Taylor et al., 2019). Further, mothers in England have reported receiving information which "pushed breastfeeding" (Burns et al., 2010). Such dictative informational transfers have been perceived as unhelpful and even harmful by mothers (Taylor et al., 2019; Burns et al., 2010), meaning information in itself is not necessarily supportive.

2. Emotional support is often expressed as empathy and connectedness between the supporter and the mother, which may or may not be related to infant feeding. Such support may signal the strength and availability of practical support mothers can draw on in future. Where mothers have a desire to breastfeed, knowing that "help is available" may promote breastfeeding by lowering the perceived opportunity costs of breastfeeding (Tully and Ball, 2013). Indeed, numerous studies on Western populations suggest emotional support is greatly valued by mothers, and seems to coexist with the perception of being able to rely on someone for support (Fox et al., 2015; Negron et al., 2013; Schmied et al., 2011). In contrast, the lack of emotional support may act as a barrier in accessing other forms of support. For example, in one qualitative study in England, lack of empathy from health professionals led to mothers being hesitant about asking for practical support (Fox et al., 2015).

3. Practical support involves direct action, for instance helping with childcare or providing financial assistance, which is likely to influence the costs and benefits of breastfeeding (Tully and Ball, 2013). Importantly, practical support is theorised to increase or decrease breastfeeding, depending on the local ecology and type of activity (Emmott and Mace, 2015; Emmott et al., 2019; Tully and Ball, 2013). Complementary support activities, such as helping with household labour, is thought to remove the need for mothers to carry out such activities, allowing mothers to invest more time and energy into breastfeeding. In contrast, conflicting support activities are those which clash with breastfeeding, thereby increasing the opportunity costs around breastfeeding. For example, high levels of infant care has been theorised and described to conflict with breastfeeding, which may create incentives for mothers to stop breastfeeding (Emmott and Mace, 2015; Lavender et al., 2006). In the UK Millennium Cohort Study, proxies of practical support from fathers and grandmothers, including paternal caregiving, have been associated with shorter breastfeeding duration (Emmott and Mace, 2015).

Overall, there is theoretical grounding and increasing indirect evidence to predict that the different types of support from different individuals may have different effects on breastfeeding. Consequently, examining the characteristics of the wider support system around the mother, rather than looking at individual support, could improve our understanding of how social support influences breastfeeding outcomes. For breastfeeding support initiatives to effectively address "unmet feeding goals" in the UK, a holistic understanding of the postnatal support around mothers could be crucial.

\subsection{Current study}

The aim of the current study is to improve understanding of the characteristics and consequences of the wider support around mothers in the UK. Specifically, using data from a convenience-sampled retrospective online survey, we explore the typologies of postnatal support and their associations with breastfeeding. We focus on support in the first few weeks after birth; a crucial period where mothers require high levels of support (Negron et al., 2013) which may be particularly important for breastfeeding outcomes (Hoddinott et al., 2011). We examine their associations with breastfeeding at two months $(2 \mathrm{~m})$, which is Public Health England's Key Performance Indicator for breastfeeding (Public Health England, 2018a). Importantly, we extend the typical view of key supporters to include family, friends and health professionals, and distinguish between the different types of support (informational, emotional and practical support).

\section{Methods}

\subsection{Survey development and data collection}

Here, we use data from a retrospective online survey. The survey was developed as part of a wider project on social support and maternal experience (https://osf.io/7kb5q/), hosted on Opinio (survey platform; http://objectplanet.com/opinio/). The final survey included questions on participant characteristics, household characteristics, child characteristics, birth experiences, support experiences and infant feeding experiences, taking 15-20 min to complete. An earlier version of the survey was independently tested and reviewed by two women unrelated to the project, who did not take part in the final survey. For more information on the survey, see https://osf.io/dbtpy/.

Women were eligible to take part in the survey if they currently lived and last gave birth in the UK, and their youngest child was under 24 months old at the time of the survey. As an exploratory study, we took an opportunistic approach and recruited participants through convenience-sampling between December 2017 and February 2018. While convenience-sampling is likely to introduce recruitment bias, it is cost and time efficient (Etikan et al., 2015). Survey adverts were posted on Twitter and Facebook (social networking sites), as well as Netmums (forum-based parenting website). Specifically for Facebook, survey adverts were posted on parenting-related Facebook groups with the permission of group administrators. The Facebook groups were diverse, including parenting groups, infant feeding support groups, and secondhand baby-item groups. Note, studies have shown that social media survey recruitment can lead to an increased proportion of middle-class participants (Topolovec-Vranic and Natarajan, 2016); however, this trend is not consistent, and it can be an effective way to recruit "hardto-reach" populations (Topolovec-Vranic and Natarajan, 2016). We specifically targeted local Facebook groups based around the UK in an attempt to diversify our sample.

Survey adverts did not explicitly mention breastfeeding, but focused on support for new mothers and maternal experience (see supplementary information; SI). On the survey landing page, participants were informed that there would be some questions about infant feeding, with an explicit statement that it did not matter whether infants were breastfed or formula fed. While the survey could be accessed via mobile phone devices, participants were informed that it may be easier to take the survey on a personal computer/laptop. Multiple-entries were prevented using IP-address checks. Overall, 701 eligible mothers took part in the survey. Ethical approval for the survey was obtained from the UCL Research Ethics Committee (ref: 11479/001).

\subsection{Analysis sample}

For the current study, we explore postnatal support around mothers and its association with breastfeeding at $2 \mathrm{~m}$. We therefore restrict our 
sample to those with breastfeeding information at 2 months (162 cases without breastfeeding information at 2 months removed from sample, including mothers whose infants were under 2 months old at time of survey). We further restrict our sample to mothers whose youngest child is a singleton (6 multiple birth cases removed) due to theoretical considerations, in that the support needs and pathways for multiple births may be different. This reduced our final eligible sample to $\mathrm{n}=533$. Data from our analyses for participants in our eligible sample who consented to publicly share their data are available online ( $=526$; https://osf.io/7kb5q/).

\subsection{Key variables used in the current study}

\subsubsection{Postnatal support}

Mothers were asked to retrospectively report whether they had received various types of support in the "first few weeks after birth." For practical/instrumental support, we asked, "Thinking back to the first few weeks after giving birth to your youngest child(ren), did the people listed below do any of the following things regardless of how helpful it was? Please tick all that apply." Specifically, we asked mothers if their partner, mother (maternal grandmother), father (maternal grandfather), brother (s), sister(s), partner's mother, partner's father and friends "did housework/chores around the house", "fed my baby", "generally looked after my baby." Note, we asked mothers to report behaviours regardless of how helpful it was, in order to minimise reporting bias.

For informational support, we asked whether mothers received advice and/or information on "looking after my baby" and "breastfeeding". In addition to the family and friends listed above, we asked whether mothers had received this information from several healthrelated professionals which included doctors (general practitioners), midwives, health visitors and breastfeeding mentors/peer supporters. In the UK, midwives are specialist antenatal-care nurses, engaging with mothers throughout pregnancy, during delivery, and soon after birth. Health visitors are specialist community public health nurses whose responsibilities include providing home visits, usually staring shortly before birth and continuing a few times until the child reaches age two - although there is variation in service provisions between geographic regions (Public Health England, 2018b). While breastfeeding mentors and peer support services are diverse, they are usually commissioned or voluntary supporters in the community who are specifically trained to provide breastfeeding information, advice, and support (The Breastfeeding Network, 2018).

Finally, for emotional support, we asked, "Thinking back to the first few weeks after giving birth to your youngest children, overall, how emotionally supported did you feel by the following people?" and asked how supported mothers felt by all the family members, friends, and health professionals mentioned above on a 5-point scale of "Very supported", "Supported", "Neither supported nor unsupported", "Unsupported" and "Very unsupported." For our analyses, "Very supported" and "Supported" were coded as receiving support, and "Neither Supported nor Unsupported", "Unsupported", and "Very unsupported" were coded as not receiving support.

For all support items, if participants selected "Not Applicable," we interpreted this as an active indication that they did not receive that particular type of support, and was therefore coded as not receiving support. Non-response was treated as missing and removed from our analyses.

\subsubsection{Breastfeeding}

Mothers were asked if they had ever breastfed the focal child (i.e. their youngest), and if so, how long for. This was used to derive two binary variables; ever breastfed, capturing breastfeeding initiation of the focal child, and any breastfeeding at 2 months, indicating whether or not the mother was providing any breastmilk to the focal child at two months of age. 2 months was chosen as a cut-off point to reflect Public Health England's Key Performance Indicator, measuring breastfeeding in any form for 6-8 weeks (Public Health England, 2018a). Note, all mothers in our sample had initiated breastfeeding; likely a consequence of our convenience-sampling, discussed further in our limitations.

\subsubsection{Socio-demographics}

In terms of family demographics, we present information on the mother's age at birth of the focal child, the focal child's sex, ethnicity, number of siblings, and mother's partnership status. As a measure of socio-economic position, we asked mothers about their highest qualification based on the UK education system. "Secondary Education" captures middle- and high-school qualifications including GCSEs, AS/ALevels or equivalent (including O-Levels, Level 1-5 awards, Advanced Apprenticeships, International Baccalaureate, National Certificates and National Diplomas). "Higher Education" includes graduate degrees or equivalent (including graduate certificates and Level 6 awards). "Postgraduate Education" includes postgraduate degrees or equivalent (including PGCEs, Doctorates, Master's and Level 7-8 awards). If none of these applied, mothers could select "Other", "Prefer not to say" or "No qualification." As a measure of subjective socio-economic experience, mothers were also asked, "How would you describe your current financial situation?". This was measured on a 5-point scale of "living comfortably", "doing alright", "just about getting by", "finding it quite difficult", and "finding it very difficult".

\subsection{Analysis}

We first provide information of our sample characteristics, including descriptive statistics on the different types of postnatal support from different individuals reported by the mothers. We then present results from our latent class regression, an extension of latent class analyses, to explore the typologies of postnatal support and how this is associated with breastfeeding at 2 months in our sample of UK mothers. Latent class analyses can be used to identify how individuals cluster into discreet groups based on available data (Hagenaars and McCutcheon, 2002), and the patterns in the clustering can be used to infer the characteristics of the different classes (i.e., typologies).

\subsubsection{Latent class regression procedure}

We began with a descriptive exploration of the data alongside exploratory latent class analyses to determine the support items and the number of classes to include in our final latent class regression model (see SI). Based on various model fit statistics and our subjective assessment of typology structures, our exploratory analyses led to a final model with three latent classes derived from practical, informational, and emotional support items from partners, mother's mothers (maternal grandmothers) and partner's mothers, as well as informational and emotional support items from friends, midwives and health visitors. Note, all support items from grandfathers, siblings, doctors, and breastfeeding supporters/mentors, as well as practical support items from friends, were removed from the final analyses as they did not improve model fit based on a range of indicators. A detailed outline of these exploratory analyses is provided in the SI.

Once the class numbers and key support items were identified, we conducted a "one-step" latent class regression model to explore how different typologies of postnatal support were associated with breastfeeding at 2 months. "One-step" latent class regression models are similar to running multinomial regressions with latent classes as the outcome, but the latent classes themselves are estimated simultaneously as the regression model (Bolck et al., 2004). In our preliminary analyses, we carried out a series of latent class regressions to select predictor variables for inclusion in our final model based on model fit (see SI).

Our final latent class regression included breastfeeding at 2 months, focal child's sex, and number of focal child's siblings as predictors, with the three latent classes as the outcome variable. All analyses were conducted in $\mathrm{R} v$ 3.5.2. All latent class analyses were performed using the $\mathrm{R}$ 
Table 1

Sample characteristics of our full eligible sample and final model sample.

\begin{tabular}{|c|c|c|c|c|c|c|c|}
\hline Full analysis sample, $\mathrm{N}=533$ & Mean & Range & SD & Final model sample, $\mathrm{N}=432$ & Mean & Range & SD \\
\hline Mother's age at birth of focal child & 32.3 & $18-44$ & 4.4 & Mother's age at birth of focal child & 32.5 & $18-44$ & 4.25 \\
\hline \multirow[t]{2}{*}{ Number of focal child's (older) siblings at time of survey } & 0.44 & $0-4$ & 0.63 & Number of focal child's (older) siblings at time of survey & 0.44 & $0-4$ & 0.62 \\
\hline & $\mathrm{N}$ & $\%$ & & & $\mathrm{~N}$ & $\%$ & \\
\hline Ever breastfed focal child (breastfeeding initiation) & & & & Ever breastfed focal child (breastfeeding initiation) & & & \\
\hline Yes & 533 & 100 & & Yes & 432 & 100 & \\
\hline No & 0 & 0 & & No & 0 & 0 & \\
\hline Any breastfeeding at 2 months & & & & Any breastfeeding at 2 months & & & \\
\hline Yes & 442 & 82.9 & & Yes & 359 & 83.1 & \\
\hline No & 91 & 17.1 & & No & 73 & 16.9 & \\
\hline Partner status ${ }^{\mathrm{a}}$ & & & & Partner status & & & \\
\hline Partnered with biological father of focal child & 517 & 97.0 & & Partnered with biological father of focal child & 428 & 99.1 & \\
\hline Partnered, not the biological father of focal child & 4 & 0.8 & & Partnered, not the biological father of focal child & 4 & 0.9 & \\
\hline Currently not partnered & 12 & 2.3 & & Currently not partnered & 0 & 0 & \\
\hline Focal child sex & & & & Focal child sex & & & \\
\hline Male & 262 & 49.2 & & Male & 217 & 49.8 & \\
\hline Female & 271 & 50.8 & & Female & 215 & 50.2 & \\
\hline Focal child ethnicity & & & & Focal child ethnicity & & & \\
\hline White & 495 & 92.9 & & White & 407 & 94.2 & \\
\hline Other & 38 & 7.1 & & Other & 25 & 5.8 & \\
\hline Mother's Highest Qualification & & & & Mother's Highest Qualification & & & \\
\hline Secondary Education & 98 & 18.4 & & Secondary Education & 72 & 16.7 & \\
\hline Higher Education & 201 & 27.7 & & Higher Education & 158 & 36.6 & \\
\hline Postgraduate Education & 230 & 43.2 & & Postgraduate Education & 199 & 46.1 & \\
\hline Other/Unknown & 4 & 0.8 & & Other/Unknown & 3 & 6.9 & \\
\hline Financial Situation & & & & Financial Situation & & & \\
\hline Living comfortably/doing alright & 339 & 63.6 & & Living comfortably/doing alright & 315 & 72.9 & \\
\hline Just about getting by & 92 & 17.3 & & Just about getting by & 84 & 19.4 & \\
\hline Finding it quite/very difficult & 30 & 5.6 & & Finding it quite/very difficult & 25 & 5.8 & \\
\hline Missing (inc. Prefer not to say) & 72 & 13.5 & & Missing (inc. Prefer not to say) & 8 & 1.9 & \\
\hline Maternal employment (at time of survey) ${ }^{\mathrm{a}}$ & & & & Maternal employment (at time of survey) ${ }^{\mathrm{a}}$ & & & \\
\hline Employed & 427 & 73.7 & & Employed & 371 & 85.9 & \\
\hline Not employed & 79 & 13.6 & & Not employed & 59 & 13.7 & \\
\hline Missing & 73 & 12.6 & & Missing & 2 & 0.5 & \\
\hline
\end{tabular}

a These percentages do not add up to 100 due to rounding.

package poLCA $v$ 1.4.1 (Linzer and Lewis, 2011). In our exploratory analyses, some of our variable selection steps were performed using the R package LCAvarsel v.1.1 (Fop and Murphy, 2017) (see SI).

\section{Results}

\subsection{Sample characteristics}

Table 1 outlines the sample characteristics of our full eligible sample ( $\mathrm{N}=533$ ) and our final model sample with complete responses ( $\mathrm{N}=432$ after listwise deletion). Most mothers in our sample had a partner at the time of the survey, who was the biological father of their child (full sample $=97 \%$; final model sample $=99.1 \%$ ). A high proportion of mothers reported post-graduate education (full sample $=43.2 \%$; final model sample $=46.1 \%$ ). In both samples, all mothers reported breastfeeding initiation, and a high proportion of mothers reported breastfeeding at 2 months (full sample $=82.9 \%$, final model sample $=83.1 \%$ ). This suggests there is an over-representation of breastfeeding mothers from higher socio-economic positions in our sample, likely due to our convenience-sampling method.

Table 2 outlines the proportion of mothers who reported receiving different types of support from different individuals, for our full sample and final model sample. In our data, partners and maternal grandmothers were most likely to be reported as providing practical and emotional support. For example, in our full eligible sample, $97.4 \%$ of fathers and $61.7 \%$ of maternal grandmothers were reported to have done housework/chores around the house, while $94.7 \%$ of fathers and $79.1 \%$ of maternal grandmothers were reported to have provided emotional support. Informational support was most likely to be reported from specialist nurses, with $81.5 \%$ of midwives and $72.1 \%$ of health visitors reported to have provided information on breastfeeding.

\subsection{Typologies of postnatal support}

Here we present results from our final model; a three-class latent class regression including any breastfeeding at 2 months, child's sex and number of siblings as predictors of class membership. Fig. 1 shows the estimated probability of receiving support for each support type and source for each class $(\mathrm{N}=432$ ). Darker (green) colours indicate a relatively high probability of support, while lighter (yellow) colours indicate a relatively low probability of support. For example, mothers in Class 1 have a relatively low probability reporting infant feeding support from partners and grandmothers $(P=0.25$ and $P=0.009$, respectively), whereas mothers in Class 2 have a relatively high probability of reporting infant feeding support from partners and grandmothers ( $\mathrm{P}=1$ and $\mathrm{P}=0.76$, respectively).

Based on this distribution of support across the classes, we constructed descriptions of the latent classes identified in our 3-class model (Table 3). We name Class 1 as the extensive support group, where mothers are likely to receive postnatal support from all individuals, but with low probability of help with infant feeding by the supporters. Class 2 is named the family support group, where mothers are likely to receive high support from partners and grandmothers, including help with infant feeding. However, mothers in this group are less likely to report receiving support from health professionals, particularly emotional support. Finally, Class 3 is named the low support group, where mothers are less likely to receive support across all the different supporters, particularly from their own mothers.

\subsection{Characteristics associated with the typologies of postnatal support}

Table 4 outlines how breastfeeding at 2 months, focal child's sex and number of focal child's siblings are associated with the typologies of postnatal support in our final model. Breastfeeding at 2 months was 


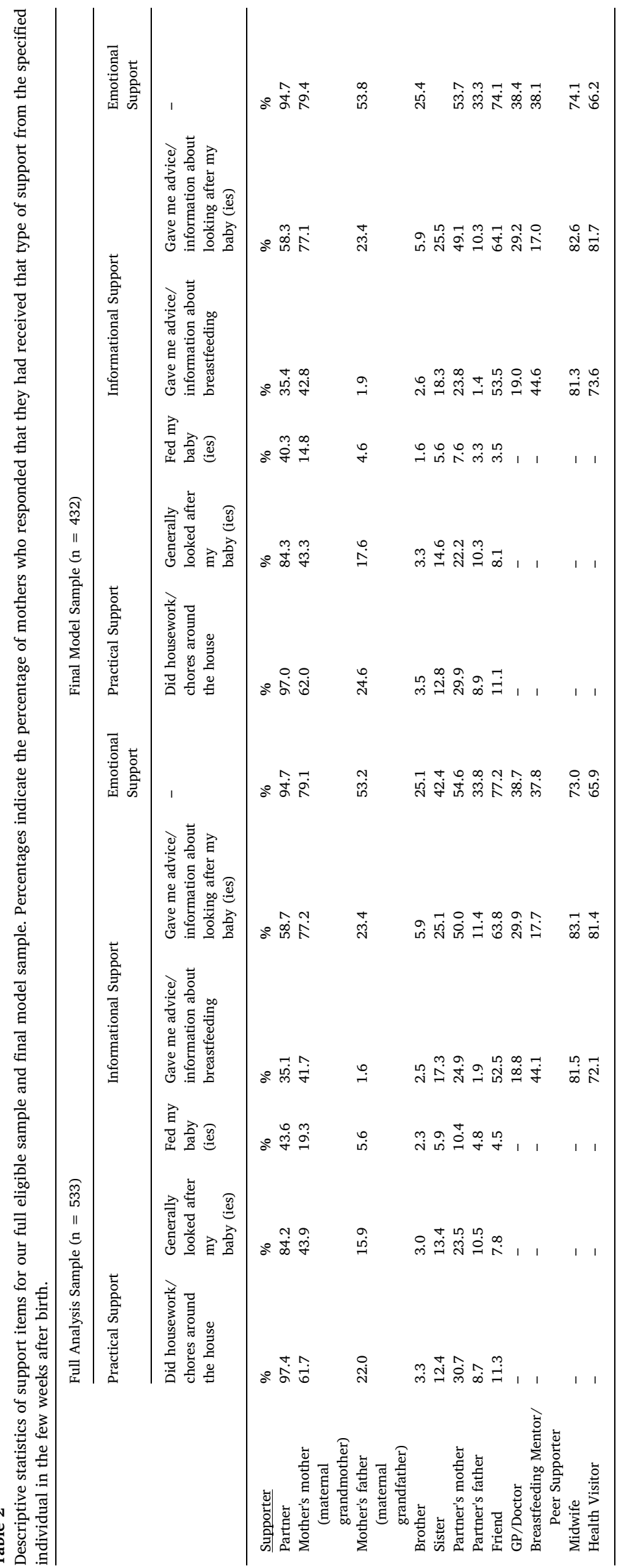




\begin{tabular}{|c|c|c|c|c|c|c|}
\hline \multirow{2}{*}{3 Class Model ( $\mathrm{N}=432$ ) } & \multirow{2}{*}{\multicolumn{3}{|c|}{ Partner }} & \multirow{2}{*}{\multicolumn{3}{|c|}{ Friend(s) }} \\
\hline & & & & & & \\
\hline Support Type & Class 1 & Class 2 & Class 3 & Class 1 & Class 2 & Class 3 \\
\hline \multirow{2}{*}{$\begin{array}{r}\text { Chores around the house } \\
\text { Looked after baby }\end{array}$} & 1 & 0.9744 & 0.9235 & & -- & -- \\
\hline & 0.8974 & 0.9364 & 0.7105 & -- & -- & -- \\
\hline \multirow{6}{*}{$\begin{array}{r}\text { Fed baby } \\
\text { Breastfeeding information } \\
\text { Caregiving information } \\
\text { Emotional support }\end{array}$} & 0.2502 & 1 & 0.2949 & -- & -- & -- \\
\hline & 0.4615 & 0.3708 & 0.188 & 0.7241 & 0.5105 & 0.2714 \\
\hline & 0.7308 & 0.6013 & 0.3578 & 0.8235 & 0.6861 & 0.3499 \\
\hline & 0.992 & 0.9244 & 0.893 & 0.8992 & 0.6652 & 0.6627 \\
\hline & Mother's I & Mother & & Midwife(u & ives) & \\
\hline & Class 1 & Class 2 & Class 3 & Class 1 & Class 2 & Class 3 \\
\hline \multirow{2}{*}{$\begin{array}{r}\text { Chores around the house } \\
\text { Looked after baby }\end{array}$} & 0.7466 & 0.8556 & 0.3056 & -- & -- & -- \\
\hline & 0.5407 & 0.759 & 0.0947 & -- & -- & -- \\
\hline \multirow{6}{*}{$\begin{array}{r}\text { Fed baby } \\
\text { Breastfeeding information } \\
\text { Caregiving information } \\
\text { Emotional support }\end{array}$} & 0.0089 & 0.7594 & 0.0131 & -- & -- & -- \\
\hline & 0.6684 & 0.5104 & 0.0317 & 0.9133 & 0.7729 & 0.6871 \\
\hline & 0.9862 & 0.9561 & 0.3535 & 0.915 & 0.7539 & 0.7371 \\
\hline & 0.9632 & 0.9214 & 0.4761 & 0.8322 & 0.6016 & 0.6841 \\
\hline & Partner's I & Mother & & Health Vis & tor(s) & \\
\hline & Class 1 & Class 2 & Class 3 & Class 1 & Class 2 & Class 3 \\
\hline \multirow{2}{*}{$\begin{array}{r}\text { Chores around the house } \\
\text { Looked after baby }\end{array}$} & 0.3122 & 0.3608 & 0.2442 & -- & -- & -- \\
\hline & 0.2393 & 0.3291 & 0.138 & -- & -- & -- \\
\hline \multirow{4}{*}{$\begin{array}{r}\text { Fed baby } \\
\text { Breastfeeding information } \\
\text { Caregiving information } \\
\text { Emotional support }\end{array}$} & 0 & 0.3303 & 0.0474 & -- & -- & -- \\
\hline & 0.2829 & 0.3056 & 0.1362 & 0.8503 & 0.6476 & 0.6182 \\
\hline & 0.5903 & 0.5623 & 0.3056 & 0.8896 & 0.8039 & 0.7185 \\
\hline & 0.6038 & 0.5509 & 0.4318 & 0.7393 & 0.6339 & 0.5647 \\
\hline
\end{tabular}

Fig. 1. Predicted probability of support for each support item by class, from our final 3-class latent class regression ( $\mathrm{n}=432$ ).

associated with a relative risk ratio (RRR) of 0.008 for being in the family support group compared to the extensive support group (95\% $\mathrm{CI}=0.001,0.104 ; p<0.001$ ), which means that women who breastfed for 2 months or longer were $99.2 \%$ less likely to be in the family support group compared to the extensive support group. Similarly, breastfeeding at 2 months was associated with a relative risk ratio of 0.057 for being in the low support group compared to the extensive support group (95\% $\mathrm{CI}=0.005,0.616 ; p=0.019$ ), which means women who breastfed for 2 months or longer were $94.3 \%$ less likely to be in the low support group compared to the extensive support group. Fig. 2 displays the predicted probabilities of mothers' postnatal support typology by breastfeeding duration. Among mothers who breastfed for 2 months or longer, 59\% were predicted to have extensive support, compared to $9 \%$ with family support, and $32 \%$ with low support. For mothers who breastfed for less than 2 months, $4 \%$ were predicted to have extensive support, compared to $62 \%$ with family support and $34 \%$ with low support. Overall, based on our model estimates, $94 \%$ of women with extensive support are predicted breastfeed for 2 months or longer, followed by $48 \%$ in the low support group and $13 \%$ in the family support group.
Finally, our results also indicated that mothers were less likely to be in the low support group, compared to the extensive support group, if the focal child was male $(\mathrm{RRR}=0.504 ; 95 \% \mathrm{CI}=0.292,0.871$; $\mathrm{p}=0.015$ ), and more likely to be in the low support group when there were more siblings in the household $(\mathrm{RRR}=2.120 ; 95 \% \mathrm{CI}=1.371$, 3.276; $\mathrm{p}=0.001$ ).

\section{Discussion}

Overall, mothers in our sample reported a wide range of postnatal support from different individuals. However, they were most likely to report practical and emotional support from fathers and maternal grandmothers, and informational support from health professionals. In our data, we find evidence of three distinct typologies of postnatal support: 1) mothers with extensive support were likely to report support from all supporters and across support types, bar infant feeding; 2) mothers with family support were likely to report support from family, including infant feeding, but were less likely to receive support from health professionals compared to mothers with extensive support; and 3)

Table 3

Typologies of social support. Descriptions of classes identified in our final 3-class latent class regression model.

\begin{tabular}{|c|c|c|c|}
\hline & $\begin{array}{l}\text { Predicted class } \\
\text { membership }\end{array}$ & $\begin{array}{l}\text { Proposed typology } \\
\text { name }\end{array}$ & Typology characteristics \\
\hline Class 1 & 0.493 & $\begin{array}{l}\text { Extensive support } \\
\text { group }\end{array}$ & $\begin{array}{l}\text { Relatively high probability of postnatal support from all supporters, apart from practical support regarding infant } \\
\text { feeding. }\end{array}$ \\
\hline Class 2 & 0.178 & Family support group & $\begin{array}{l}\text { Relatively high probability of support from family members (partner and grandmothers), including practical } \\
\text { support with infant feeding. Relatively lower probability of support from health professionals, particularly } \\
\text { emotional support. }\end{array}$ \\
\hline Class 3 & 0.329 & Low support group & $\begin{array}{l}\text { Relatively low support across all supporters, particularly maternal grandmothers, with most likely support being } \\
\text { practical and emotional support from the partner. }\end{array}$ \\
\hline
\end{tabular}


Table 4

Latent Class Regression results, similar in interpretation as a multinomial logistic regression. RRR $=$ relative risk ratio.

\begin{tabular}{|c|c|c|c|c|c|c|c|c|}
\hline \multirow[t]{2}{*}{$N=432$} & \multicolumn{4}{|c|}{$\begin{array}{l}\text { Family support group (Allen and Hector, 2005), compared to } \\
\text { Extensive support group (0) }\end{array}$} & \multicolumn{4}{|c|}{$\begin{array}{l}\text { Low support group (Allen and Hector, 2005), compared to Extensive } \\
\text { support group (0) }\end{array}$} \\
\hline & $\mathrm{b}$ & se & RRR & RRR 95\% CI & $\mathrm{b}$ & se & RRR & RRR 95\% CI \\
\hline Intercept & 2.853 & 1.232 & 17.3 & $1.404,214.2$ & 2.249 & 1.215 & 9.48 & $0.876,102.5$ \\
\hline $\begin{array}{l}\text { Any breastfeeding at } 2 \text { months } \\
\quad \text { (ref. }=\text { no) }\end{array}$ & -4.791 & 1.288 & 0.008 & $0.001,0.104$ & -2.861 & 1.213 & 0.057 & $0.005,0.616$ \\
\hline Child sex (ref. $=$ female) & 0.340 & 0.430 & 1.406 & $0.605,3.264$ & -0.684 & 0.279 & 0.504 & $0.292,0.871$ \\
\hline Number of focal child's siblings & -0.397 & 0.370 & 0.673 & $0.325,1.390$ & 0.751 & 0.222 & 2.120 & $1.371,3.276$ \\
\hline
\end{tabular}

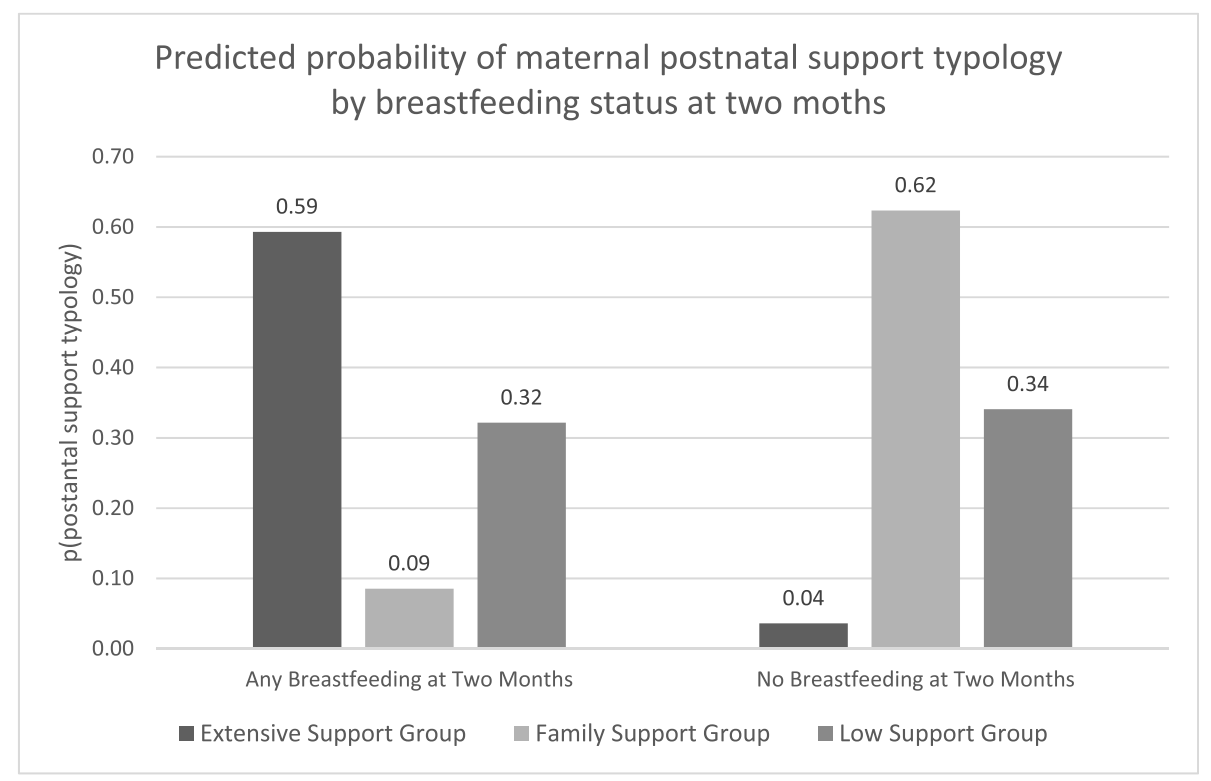

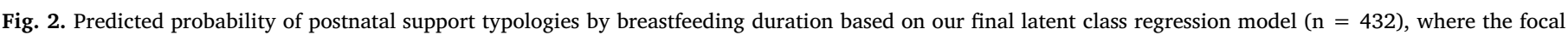
child is female and there are no other children (focal child's siblings) in the household.

mothers with low support were less likely to report support across all supporters and support types. Mothers who breastfed for $2 \mathrm{~m}$ or longer were most likely to receive extensive support (59\%), followed by low support $(32 \%)$ and family support $(9 \%)$. It is important to note that these results are derived from a sample of mothers who all initiated breastfeeding, with a large proportion of mothers in higher socio-economic positions. Further, the causal directions between the typologies of postnatal support and breastfeeding for $\geq 2 \mathrm{~m}$ are unknown. Nonetheless, our findings demonstrate the potential for diversity in support experiences, even within a fairly homogenous group of UK mothers.

\subsection{The importance of extensive support}

Our results highlight the complex pathways between social support and breastfeeding: Compared to those with low support, receiving support from family may be associated with both an increased and decreased risk of early breastfeeding cessation - depending on whether family members provided practical infant feeding support. Mothers with extensive support reported very little infant feeding by others, while mothers with family support had a high probability of infant feeding by family. Such practical infant feeding support, often requiring introduction of the bottle, is likely to increase the probability of early breastfeeding cessation: Introduction of artificial nipples, bottle feeding of formula, as well as "exclusive" bottle feeding of breastmilk have all been associated with breastfeeding cessation (Fein et al., 2008; Jiang et al., 2015; Howard et al., 2003). Mechanistically, the introduction of the bottle/artificial nipples are associated with development of different sucking patterns in infants (Moral et al., 2010), and sucking patterns (both in infants and breast pumps) can influence milk production (Meier et al., 2011). While the causal direction between infant feeding by family and breastfeeding cessation is unclear due to the retrospective nature of our data, our findings reflect the importance of distinguishing the different types of support behaviours and how they relate to breastfeeding outcomes, adding to previous findings (Emmott and Mace, 2015; Cisco, 2017).

A further key difference between extensive and family support was the prevalence of informational and emotional support from health professionals - with a higher likelihood of professional support in the extensive support group associated with both lower help with infant feeding by family, and higher probability of breastfeeding at $2 \mathrm{~m}$. Thus, our results also highlight the importance of professional support in the $\mathrm{UK}$, where the weakened breastfeeding culture may mean mothers are particularly dependent on professionals for informational and emotional support. For example, for mothers who have access to family support, support from health professionals (thereby experiencing extensive support) may facilitate family members to support mothers without engaging with infant feeding, for instance by giving mothers the tools to direct family support to other activities. At the same time, lack of adequate professional support may mean mothers are not able to access or assess useful advice and information on how to breastfeed, feel confident about breastfeeding, and overcome breastfeeding challenges. To illustrate our point, in our open-text question at the end of our survey, one participant recounted her stressful experience of trying to seek professional support after experiencing challenges with breastfeeding - and, while she was supported by her family and friends, she 
was ultimately unable to continue after a month of pumping:

During pregnancy I assumed I would breastfeed and that it wouldn't be a problem. [Unfortunately] I found it difficult, my baby had problems latching and she kept losing weight after the first week before we were advised to combine with formula feeds. I sought help to improve latch from healthcare professionals but received little or no useful advice. In the worst cases I was given conflicting advice which may have contributed to the weight loss. With a lot of family and friend support I pumped for the first month and combined with formula to give my baby 'the best'. ... I felt unsupported and enormous guilt, I still do 5 months on!

It is important to note that the causal directions between these findings are unclear. For example, maternal postnatal support may change into a family-oriented system after early breastfeeding cessation (i.e., early breastfeeding cessation leading to the family support typology). Equally, practical infant feeding support from family may encourage breastfeeding cessation (i.e., family support typology leading to breastfeeding cessation). Nonetheless, these results reiterate why an individual behaviour-change approach to improving breastfeeding rates, exclusively targeting mothers, may not be particularly effective in the UK. In our sample of mothers, a range of family members and friends were providing different types of postnatal support. However, as family and friends are often not present when mothers receive professional breastfeeding support, many may lack the skills or knowledge to effectively support maternal breastfeeding goals (Hoddinott and Pill, 1999; Fox et al., 2015; Grassley and Eschiti, 2008). Previous studies have indicated that mothers require and value support from family and friends (Negron et al., 2013; Grassley and Eschiti, 2008), and that they are important determinants of breastfeeding outcomes (Clifford and McIntyre, 2008) - which, from an evolutionary perspective, influences the costs and benefits around breastfeeding behaviour (Emmott and Mace, 2015). In order to create extensive support for mothers, professional supporters may therefore need to engage with the wider support network around mothers. Our findings add evidence to support recent public health policy movements from targeting individuals to a familycentred approach, which are particularly gaining momentum in the UK regarding early-years universal support and interventions (House of Commons Health and Social Care Committee, 2019). However, the "family" in family-centred approaches are often limited to the nuclear family, namely mothers and their partners. Our results highlight the potential for looking "beyond the nuclear family", in particular by including maternal grandmothers who were the largest providers of practical and emotional support after partners.

\subsection{Low support and breastfeeding}

In our final model, we also found that mothers in the low support group were less likely to breastfeed for 2 months or longer compared to the extensive support group, but more likely to breastfeed compared to the family support group. Given that social support is a known determinant of breastfeeding, it is puzzling to find that the probability of early breastfeeding cessation is lower for mothers receiving low support compared to mothers receiving family support. One possibility is that some mothers with low support did not "require" support to maintain breastfeeding. To exemplify this, one participant told us:

My husband has supported me greatly and the reason our parents and friends have not supported us massively is because luckily everything has been very straightforward for us and I have not really needed much support. Having a child is relentless and draining and so having my friends listen to me moan about being tired is about all the support I have needed.

This could also explain why higher parity (i.e., high number of focal child's siblings) predicted low support in our final model: Some mothers with previous experiences of infant feeding may have had more knowledge and skill to maintain breastfeeding without having to draw on much support from family, friends and health professionals. Several participants told us how their experiences from their first child meant they required less support, including one participant who wrote:

I feel as this was my second child I had a greater confidence and less support was needed - had I been answering after the birth of my first child the answers may have been very different ...

However, given that low support is associated with a higher probability of early breastfeeding cessation compared to extensive support, we hypothesise that our low support group is a heterogeneous typology which also includes women who required but did not receive adequate support from their social network and professionals. Indeed, some of our participants recounted their often heart-breaking experiences of not receiving the support they needed. For example:

My experience of postnatal care and infant feeding support were so completely negative I am still struggling to process it. It was not just negative, but bullying to the point of abusive. At my most vulnerable, I felt pressurised, bullied, manipulated and humiliated by institutions I trusted, such as the NHS and the NCT. I was made to feel that my mental health was not only unimportant but in fact necessarily expendable for the sake of my baby. My own self-destructive and self-punishing instincts were enabled to the point of my baby starving and my developing serious postnatal depression. I was convinced I was the world's worst mother by the time my daughter was only a few weeks old.

Our results also indicated that mothers with female infants were more likely to be in the low support group compared to mothers with male infants. The sex ratio of children in our sample was relatively even at 101 (49.8\% girls, $50.2 \%$ boys), meaning this result is not an artefact of a sex-ratio skew. While the mechanisms around this finding is unclear, both biological mechanisms and social norms in the UK may encourage allomothers to provide more support to mothers with male infants: Biologically, mothers with male infants produce more breastmilk, and produce breastmilk with greater nutritional content, compared to mothers with female infants (Powe et al., 2010). Giving birth to boys is also associated with increased risk of obstetric complications (Brettell et al., 2008), pre-eclampsia (Elsmén et al., 2006), gestational diabetes (Di Renzo et al., 2007), and postnatal depression (Myers and Johns, 2019). Overall, the greater "biological costs" associated with boys could mean mothers with male infants require and receive more social support than mothers with female infants. Further, studies have suggested son-biases in fathering in the UK (Emmott and Mace, 2018) which may reflect son-biases in wider caregiving, although femalebiases in grandparental support have also been reported (Tan et al., 2010). Given the uncertainties around possible mechanisms, we caution against drawing strong conclusions regarding the association between infant sex and the typology of social support.

Overall, we hypothesise two different pathways to low support: some women may experience low support as they do not require the support, while others may experience low support as they are unable to access support. This may explain why, as a typology of postnatal support, low support is associated with a lower probability of breastfeeding for 2 months or longer compared to extensive support, but a higher probability breastfeeding for 2 months or longer compared to family support. In our current study, we are unable to explicitly test this hypothesis post-hoc, as we do not have the necessary information on the differential need for support. In future, it may be useful for researchers to attempt to distinguish between low support by unmet support needs.

\section{Limitations}

First, our data are the product of convenience-sampling, and mothers in our sample were more likely to be partnered, be from a White ethnic background, and have a higher education level than the general 
UK population. Importantly, all mothers in our final sample had initiated breastfeeding. While we hope our findings are useful in informing research with mothers from more diverse backgrounds, our current findings should not be directly extrapolated to different groups of mothers in the UK and beyond. In future, we recommend researchers carry out purposive sampling, including targeting women who did not initiate breastfeeding, or targeting women from different ethnic backgrounds.

Second, the sources of support and the types of support in our study was pre-defined, meaning we may have overlooked key supporters and support behaviours. While our survey questions were theoretically informed, the typologies we have identified are limited by the supporters and support items included in our survey. To address this issue, future studies may benefit from social network mapping and analysis, with more observational and ethnographic studies clarifying what types of support mothers actually receive in the postnatal period.

Finally, our data was collected retrospectively, meaning there is a risk that self-reported support may be coloured by breastfeeding experience. Further, we lack granular information on the frequency/ timings of postnatal support, and for mothers who stopped breastfeeding before 2 months, it is unclear whether support came before or after breastfeeding cessation. Consequently, the causal pathways between the associations we find in this study are unclear. For instance, while receiving help with infant feeding may induce breastfeeding cessation, breastfeeding cessation may also create opportunities for supporters to provide help with infant feeding. Nonetheless, our study serves as the first step in understanding the broader postnatal support networks around mothers, and we hope researchers with opportunities for prospective data collection will build on our findings.

\section{Conclusions}

Our results add to a growing body of literature highlighting the importance of family, friends and health professionals in breastfeeding outcomes (Clifford and McIntyre, 2008). Based on evolutionary anthropological theory, we predicted that long-term breastfeeding is dependent on extensive support from multiple individuals (Quinlan and Quinlan, 2008). In support, we found that mothers with extensive postnatal support from family, friends and health professionals were most likely to breastfeed for 2 months or longer, compared to those with low support or family-biased support. As such, our findings reflect the importance of a family-centred approach to support breastfeeding in the UK, and the potential in looking "beyond the nuclear family." Our results also indicate the need for the public health literature to take a more nuanced approach to social support, including consideration of the different forms of support and the differential impact they may have.

\section{Acknowledgements}

We would like to thank all our participants who took part in our survey, who took their time to share important information about their lives with us. We would also like to thank Dr Susie Schaffnit and Eve Black, who tested, reviewed and provided feedback on an earlier version of our survey. Finally, we would like to acknowledge the Medical Research Council for part-funding this research, by funding Abigail E. Page (grant number MR/P014216/1).

\section{Appendix A. Supplementary information}

Supplementary information to this article can be found online at https://doi.org/10.1016/j.socscimed.2020.112791.

\section{References}

Allen, J., Hector, D., 2005. Benefits of breastfeeding. New South Wales Public Health
Bulletin 16 (4), 42-46. https://doi.org/10.1071/nb05011.

Bolck, A., et al., 2004. Estimating latent structure models with categorical variables: onestep versus three-step estimators. Political Anal. 12 (1), 3-27. https://doi.org/10. 1093/pan/mph001.

Bosi, A.T.B., et al., 2016. Breastfeeding practices and policies in WHO European region member states. Public Health Nutr. 19 (4), 753-764. https://doi.org/10.1017/ S1368980015001767.

Brettell, R., et al., 2008. Examination of the association between male gender and preterm delivery. Eur. J. Obstet. Gynecol. Reprod. Biol. 141 (2), 123-126. https://doi.org/10. 1016/j.ejogrb.2008.07.030.

Brown, A., 2016. What do women really want? Lessons for breastfeeding promotion and education. Breastfeed. Med. 11 (3), 102-110. https://doi.org/10.1089/bfm.2015. 0175.

Brown, A., et al., 2016. Understanding the relationship between breastfeeding and postnatal depression: the role of pain and physical difficulties. J. Adv. Nurs. 72 (2), 273-282. https://doi.org/10.1111/jan.12832.

Burns, E., et al., 2010. A meta-ethnographic synthesis of women's experience of breastfeeding. Matern. Child Nutr. 6 (3), 201-219. https://doi.org/10.1111/j.1740-8709. 2009.00209.x.

Butte, N.F., King, J.C., 2005. Energy requirements during pregnancy and lactation. Public Health Nutr. 8 (7a), 1010-1027. https://doi.org/10.1079/phn2005793.

Cardenas, R.A., Major, D.A., 2005. Combining employment and breastfeeding: utilizing a work-family conflict framework to understand obstacles and solutions. J. Bus. Psychol. 20 (1), 31-51. https://doi.org/10.1007/s10869-005-6982-0.

Cisco, J., 2017. Who supports breastfeeding mothers? Hum. Nat. 28 (2), 231-253. https://doi.org/10.1007/s12110-017-9286-y.

Clifford, J., McIntyre, E., 2008. Who supports breastfeeding? Breastfeed. Rev. 16 (2), 9-19.

Di Renzo, G.C., et al., 2007. Does fetal sex affect pregnancy outcome? Gend. Med. 4 (1), 19-30. https://doi.org/10.1016/s1550-8579(07)80004-0.

$\mathrm{Du}$, J., et al., 2019. Comparison of social complexity in two independent pastoralist societies. Behav. Ecol. Sociobiol. 73 (1), 4. https://doi.org/10.1007/s00265-0182611-6.

Elsmén, E., et al., 2006. Fetal gender and gestational-age-related incidence of preeclampsia. Acta Obstet. Gynecol. Scand. 85 (11), 1285-1291. https://doi.org/10. 1080/00016340600578274.

Emmott, E.H., Mace, R., 2015. Practical support from fathers and grandmothers is associated with lower levels of breastfeeding in the UK Millennium Cohort Study. PLoS One 10 (7), e0133547. https://doi.org/10.1371/journal.pone.0133547.

Emmott, E.H., Mace, R., 2018. Why the Son-Bias in Fathering? Testing Sex-Differences in the Associations between Paternal Caregiving and Child Outcomes in England (Preprint). OSF Preprintshttps://doi.org/10.31219/osf.io/q6fpx.

Emmott, E.H., Page, A.E., 2019. Alloparenting. In: Shackelford, T., Weekes-Shackelford, V. (Eds.), Encyclopedia of Evolutionary Psychological Science. Springer, Cham. https://doi.org/10.1007/978-3-319-16999-6_2253-1.

Etikan, I., et al., 2015. Comparison of convenience sampling and purposive sampling. Am. J. Theor. Appl. Stat. 5 (1), 1-4. https://doi.org/10.11648/j.ajtas.20160501.11.

Faircloth, C., 2014. Intensive parenting and the expansion of parenting. In: Lee, E., Bristow, J., Faircloth, C., Macvarish, J. (Eds.), Parenting Culture Studies. Palgrave Macmillan UK, London, pp. 25-50.

Fein, S.B., et al., 2008. Success of strategies for combining employment and breastfeeding. Pediatrics 122 (2), S56-S62. https://doi.org/10.1542/peds.2008-1315g.

Fomon, S.J., 2001. Infant feeding in the 20th century: formula and beikost. J. Nutr. 131 (2). https://doi.org/10.1093/jn/131.2.409S. 409S-20S.

Fop, M., Murphy, T.B., 2017. LCAvarsel: Variable Selection for Latent Class Analysis. R package version 1.1. Available from: https://cran.r-project.org/package=LCAvarsel, Accessed date: 3 January 2020.

Fox, R., et al., 2015. UK women's experiences of breastfeeding and additional breastfeeding support: a qualitative study of Baby Café services. BMC Pregnancy Childbirth 15 (1), 147. https://doi.org/10.1186/s12884-015-0581-5.

Grassley, J., Eschiti, V., 2008. Grandmother breastfeeding support: what do mothers need and want? Birth 35 (4), 329-335. https://doi.org/10.1111/j.1523-536X.2008. 00260.x.

Guyer, J., et al., 2012. Mothers' breastfeeding experiences and implications for professionals. Br. J. Midwifery 20 (10), 724-733. https://doi.org/10.12968/bjom.2012.20. 10.724.

Hagenaars, J.A., McCutcheon, A.L., 2002. Applied Latent Class Analysis. Cambridge University Press, Cambridge.

Hawkins, S.S., et al., 2007. The impact of maternal employment on breast-feeding duration in the UK Millennium Cohort Study. Public Health Nutr. 10 (9), 891-896. https://doi.org/10.1017/S1368980007226096.

Hoddinott, P., Pill, R., 1999. Qualitative study of decisions about infant feeding among women in east end of London. BMJ 318 (7175), 30-34. https://doi.org/10.1136/bmj. 318.7175.30.

Hoddinott, P., et al., 2011. Global evidence synthesis and UK idiosyncrasy: why have recent UK trials had no significant effects on breastfeeding rates? Matern. Child Nutr. 7 (3), 221-227. https://doi.org/10.1111/j.1740-8709.2011.00336.x.

House of Commons Health and Social Care Committee, 2019. First 1000 Days of Life. Thirteenth Report of Session 2017-19. House of Commons (UK). Available from: https://publications.parliament.uk/pa/cm201719/cmselect/cmhealth/1496/1496. pdf, Accessed date: 3 January 2020.

Howard, C.R., et al., 2003. Randomized clinical trial of pacifier use and bottle-feeding or cupfeeding and their effect on breastfeeding. Pediatrics 111 (3), 511-518. https:// doi.org/10.1542/peds.111.3.511.

Jiang, B., et al., 2015. Evaluation of the impact of breast milk expression in early postpartum period on breastfeeding duration: a prospective cohort study. BMC Pregnancy 
Childbirth 15 (1), 268. https://doi.org/10.1186/s12884-015-0698-6.

Johnston, M.L., Esposito, N., 2007. Barriers and facilitators for breastfeeding among working women in the United States. J. Obstet. Gynecol. Neonatal Nurs. 36 (1), 9-20. https://doi.org/10.1111/j.1552-6909.2006.00109.x.

Jolly, K., et al., 2012. Systematic review of peer support for breastfeeding continuation: metaregression analysis of the effect of setting, intensity, and timing. BMJ 344, d8287. https://doi.org/10.1136/bmj.d8287.

Kramer, M.S., Kakuma, R., 2012. Optimal duration of exclusive breastfeeding. Cochrane Database Syst. Rev. 8, CD003517. https://doi.org/10.1002/14651858.CD003517. pub2.

Labbok, M.H., 2012. Global baby-friendly hospital initiative monitoring data: update and discussion. Breastfeed. Med. 7 (4), 210-222. https://doi.org/10.1089/bfm.2012. 0066.

Lavender, T., et al., 2006. Breastfeeding and family life. Matern. Child Nutr. 2 (3), 145-155. https://doi.org/10.1111/j.1740-8709.2006.00049.x.

Linzer, D.A., Lewis, J.B., 2011. poLCA: an R package for polytomous variable latent class analysis. https://doi.org/10.18637/jss.v042.i10 42, 10, 29.

Marlowe, F.W., 2003. A critical period for provisioning by Hadza men: implications for pair bonding. Evol. Hum. Behav. 24 (3), 217-229. https://doi.org/10.1016/S10905138(03)00014-X.

Martucci, J., Barnhill, A., 2018. Examining the use of 'natural' in breastfeeding promotion: ethical and practical concerns. J. Med. Ethics 44 (9), 615-620. https://doi.org/ 10.1136/medethics-2017-104455.

McAndrew, F., et al., 2012. Infant Feeding Survey 2010. Health and Social Care Information Centre. Available from: https://sp.ukdataservice.ac.uk/doc/7281/ mrdoc/pdf/7281_ifs-uk-2010_report.pdf, Accessed date: 3 January 2020.

McFadden, A., et al., 2017. Support for healthy breastfeeding mothers with healthy term babies. Cochrane Database Syst. Rev. 2, CD001141. https://doi.org/10.1002/ 14651858.CD001141.pub5.

Meier, P.P., et al., 2011. Breast pump suction patterns that mimic the human infant during breastfeeding: greater milk output in less time spent pumping for breast pump-dependent mothers with premature infants. J. Perinatol. 32, 103. https://doi. org/10.1038/jp.2011.64.

Moral, A., et al., 2010. Mechanics of sucking: comparison between bottle feeding and breastfeeding. BMC Pediatr. 10 (1), 6. https://doi.org/10.1186/1471-2431-10-6.

Myers, S., Johns, S.E., 2019. Male infants and birth complications are associated with increased incidence of postnatal depression. Soc. Sci. Med. 220, 56-64. https://doi. org/10.1016/j.socscimed.2018.10.008.

Negron, R., et al., 2013. Social support during the postpartum period: mothers' views on needs, expectations, and mobilization of support. Matern. Child Health J. 17 (4), 616-623. https://doi.org/10.1007/s10995-012-1037-4.

Page, A.E., et al., 2017. Hunter-gatherer social networks and reproductive success. Sci. Rep. 7 (1), 1153. https://doi.org/10.1038/s41598-017-01310-5.

Powe, C.E., et al., 2010. Infant sex predicts breast milk energy content. Am. J. Hum. Biol. 22 (1), 50-54. https://doi.org/10.1002/ajhb.20941.

Public Health England, 2018a. Breastfeeding Prevalence at 6-8 Weeks after Birth (Experimental Statistics). Public Health England Available online: https://assets. publishing.service.gov.uk/government/uploads/system/uploads/attachment data/ file/845709/2017-18_Annual_Breastfeeding_Statistical_Commentary.pdf, Accessed date: 3 January 2020 .
Public Health England, 2018b. Health Visitor Service Delivery Metrics. 2017/18 Annual Data Statistical Commentary. Public Health England Available from: https://assets. publishing.service.gov.uk/government/uploads/system/uploads/attachment_data/ file/750446/2017_2018_Annual_Health_Visitor_Statistical_Commentary.pdf, Accessed date: 3 January 2020

Quinlan, R.J., Quinlan, M.B., 2008. Human lactation, pair-bonds, and alloparents. Hum. Nat. 19 (1), 87-102. https://doi.org/10.1007/s12110-007-9026-9.

Schafer, E.J., et al., 2016. Social contexts of infant feeding and infant feeding decisions. J. Hum. Lactation 32 (1), 132-140. https://doi.org/10.1177/0890334415592850. 2016.

Schmied, V., et al., 2011. Women's perceptions and experiences of breastfeeding support: a metasynthesis. Birth 38 (1), 49-60. https://doi.org/10.1111/j.1523-536X.2010. 00446.x.

Scott, J., Colin, W., 2002. Breastfeeding: reasons for starting, reasons for stopping and problems along the way. Breastfeed. Rev. 10 (2), 13.

Stansfeld, S.A., et al., 2006. Social support and social cohesion. In: Marmot, M., Wilkinson, R.G. (Eds.), Social Determinants of Health, second ed. Oxford University Press, Oxford, pp. 148-171.

Tan, J.-P., et al., 2010. Filling the parenting gap? Grandparent involvement with U.K. adolescents. J. Fam. Issues 31 (7), 992-1015. https://doi.org/10.1177/ $0192513 X 09360499$.

Taylor, A.M., et al., 2019. 'Scrutinised, judged and sabotaged': a qualitative video diary study of first-time breastfeeding mothers. Midwifery 75, 16-23. https://doi.org/10. 1016/j.midw.2019.04.004.

The Breastfeeding Network, 2018. Peer Support Projects. Available from: https://www. breastfeedingnetwork.org.uk/get-involved/train-to-be-a-registered-volunteer/peersupport-programmes/, Accessed date: 3 January 2020.

Thulier, D., Mercer, J., 2009. Variables associated with breastfeeding duration. J. Obstet. Gynecol. Neonatal Nurs. 38 (3), 259-268. https://doi.org/10.1111/j.1552-6909. 2009.01021.x.

Topolovec-Vranic, J., Natarajan, K., 2016. The use of social media in recruitment for medical research studies: a scoping review. J. Med. Internet Res. 18 (11), e286. https://doi.org/10.2196/jmir.5698.

Tully, K.P., Ball, H.L., 2013. Trade-offs underlying maternal breastfeeding decisions: a conceptual model. Matern. Child Nutr. 9 (1), 90-98. https://doi.org/10.1111/j.17408709.2011.00378.x.

UNICEF, U.K., 2013. The evidence and rationale for the UNICEF UK Baby Friendly Initiative standards. Available from: https://www.unicef.org.uk/wp-content/ uploads/sites/2/2013/09/baby_friendly_evidence_rationale.pdf, Accessed date: 3 January 2020.

Victora, C.G., et al., 2016. Breastfeeding in the 21st century: epidemiology, mechanisms, and lifelong effect. The Lancet 387 (10017), 475-490. https://doi.org/10.1016/ S0140-6736(15)01024-7.

Wall-Scheffler, C.M., et al., 2007. Infant carrying: the role of increased locomotory costs in early tool development. Am. J. Phys. Anthropol. 133 (2), 841-846. https://doi. org/10.1002/ajpa.20603.

Williamson, I., et al., 2012. 'It should be the most natural thing in the world': exploring first-time mothers' breastfeeding difficulties in the UK using audio-diaries and interviews. Matern. Child Nutr. 8 (4), 434-447. https://doi.org/10.1111/j.1740-8709. 2011.00328.x. 